\title{
Effect of metamorphic overprint on early Earth isotopic compositions: the case study of Saglek Block (Labrador, Canada)
}

\author{
ADRIEN VEZINET ${ }^{1,2}$, EMILIE THOMASSOT ${ }^{3}$ AND D. \\ GRAHAM PEARSON $^{1}$ \\ ${ }^{1}$ University of Alberta \\ ${ }^{2}$ Université Grenoble Alpes \\ ${ }^{3}$ Université de Lorraine, CNRS, CRPG \\ Presenting Author: adrien.vezinet@univ-grenoble-alpes.fr
}

For the last 60 years, whole-rock isotope geochemistry has provided benchmark results that have shed light on Earth's evolution. Along with improvements in analytical resolution, those results have been complemented by spatially resolved information extracted from accessory mineral phases, predominantly zircon. Many of the results recovered from the two approaches on early Earth materials are hard to reconcile. While isotopic signatures (e.g. $\mathrm{Sm} / \mathrm{Nd}$ or $\mathrm{Lu} / \mathrm{Hf}$ ) obtained from whole-rock protocols indicate extensive chemical differentiation of the mantle before $3.6 \mathrm{Ga}$, those from spatially resolved analysis of individual minerals point toward much less dramatic differentiation, with the majority of crystal domains having subchondritic compositions.

To investigate likely reasons for the observed discrepancies between whole-rock and mineral data, we modelled the impact of contamination by an external component, onto a suite of cogenetic rocks; on both the isochron apparent age and the initial radiogenic isotope value. We show that secondary contamination can generate spurious, and resolvable, increase in initial ratio, i.e. $\left({ }^{176} \mathrm{Hf} /{ }^{177} \mathrm{Hf}\right)_{\text {initial }}$ or $\left({ }^{143} \mathrm{Nd} /{ }^{144} \mathrm{Nd}\right)_{\text {initial}}$, while apparent isochron age calculated from isochron slope remains within uncertainty of the original, undisturbed rock suite. Our results enlighten the debate about the nature of Earth's earlier mantle as they support an early Earth history that involves no significant crust-mantle differentiation before $3.6 \mathrm{Ga}$ and no $\mathrm{Hf}-\mathrm{Nd}$ isotopes decoupling at that time.

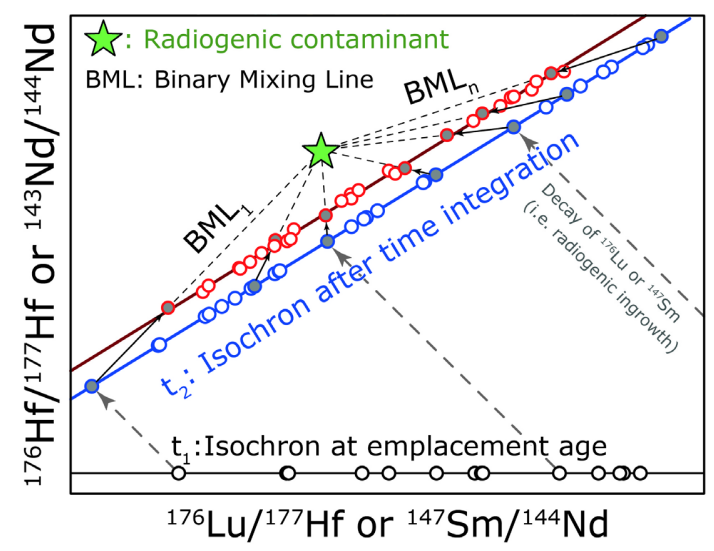

